\title{
LAS SECCIONES DISCURSIVAS EN MATEO
}

\section{Estudio de su estructura y patrones literarios}

\author{
Juan Sebastián Hernández Valencia \\ Universidad Pontificia Bolivariana, Medellín, Colombia \\ sevasteh@gmail.com \\ iD https://orcid.org/0000-0002-9567-4205
}

Resumen: Las propuestas de comprensión de la estructura literaria y teológica del evangelio de Mateo han caído en cierto subjetivismo dada la gran cantidad de recursos estilísticos del texto que el intérprete debe considerar. Entre todas las teorías, la ofrecida por Bacon desde inicios del siglo xx, con sus diferentes ampliaciones y precisiones, ha ido ganando mayor aceptación por su explicación de la unidad y centralidad de los cinco grandes discursos del evangelio. Este artículo se propone corroborar y precisar la unidad, contenido y estructura de dichas secciones discursivas analizando las fórmulas empleadas por Mateo para finalizar los cinco discursos, así como los recursos léxicos y narrativos usados al inicio de dichas secciones.

Palabras clave: Evangelio de Mateo. Secciones discursivas. Estructura narrativa. Patrones literarios. Recursos léxicos

\section{The Discoursive Sections in the Gospel of Matthew Study of Its Structure and Literary Patterns}

Abstract: The proposals for understanding the literary and theological structure of the Gospel of Matthew have fallen into a certain subjectivism given the large number of stylistic resources of the text that the interpreter must consider. Among all the theories, that offered by Bacon since the beginning of the 20th century, with its different extensions and details, has been gaining greater acceptance for its explanation of the unity and centrality of the five great discourses of the gospel. The propose of this article is to verify and specify the unity, content and structure of the discursive sections by analyzing the formulas used by Matthew to finalize the five 
speeches, as well as the lexical and narrative resources used at the beginning of such sections.

Keywords: Gospel of Matthew. Discursive sections. Narrative structure. Literary patterns. Lexical resources

\section{Introducción}

La discusión sobre la estructura del evangelio de Mateo es muy amplia ${ }^{1}$. Como señalaba Ulrich Luz en su comentario ${ }^{2}$, las numerosas y diferentes propuestas que se han presentado se pueden recoger en tres grupos: a) el pentateuco mateano (o modelo de los cinco libros); b) el modelo centro $^{3}$; y c) el modelo de división marcano ${ }^{4}$.

Por su respeto, sensibilidad y cercanía al estilo y recursos literarios del evangelio de Mateo, además de ser el más usado entre los especialistas, el pentateuco mateano es el modelo más aceptado. Este modelo fue propuesto por Benjamin Wisner Bacon en sus estudios publicados alrededor del fin de la primera guerra mundial ${ }^{5}$. Aunque, ya 65 años antes de Bacon, Delitzsch había iniciado la investigación de la composición pentateucal (pentateutische Anlage) del evangelio ${ }^{6}$. En su estudio, Delitzsch identifica los temas centrales de Mt 1,1-2,15 con el libro del Génesis, a Mt 2,16-17 con el Éxodo, a Mt 8-9 con el Levítico, Mt 10-18 con Números, y finalmente Mt 19-28 con el Deuteronomio. Si bien las diferencias entre Bacon y Delitzsch son importantes. Por ejemplo, Bacon parte de la hipótesis de la estructura de los escritos de Papías y su relación con Mateo, mientras que Delitzsch realiza un estudio temático y tipológico entre el Pentateuco y los discursos del primer evangelio.

1 Véase una síntesis de la investigación en WEREN, “Macrostructure”, 171-200; y Roig Cervera, Estructura, 2-98. Ofrecen historias de la investigación Luz, Mateo. I, 32-37; Stanton, “Origin”, 1903-1906; y NeIRYnck, “La rédaction”, 51-58. Aunque el status quaestionis presentado por estos investigadores son muy amplios, solo Neirynck habla sobre el trabajo de Franz Delitzsch, predecesor del modelo más aceptado de los cinco libros. Sobre este autor se harán algunas anotaciones más adelante.

2 Luz, Mateo. I, 34-35.

${ }^{3}$ Los representantes de esta propuesta son: Combrink, "Structure"; ElLIS, Matthew; LoHr, "Oral Techniques"; y FENTON, “Inclusio and Chiasmus”.

${ }^{4}$ Kingsbury, Matthew, 7-25.

5 Bacon, “Jesus and the Law”, 203-231; ID., Studies in Matthew, 80-90.

6 Delitzsch, Untersuchungen, 56-109. 
La idea central de los estudios de Bacon es la construcción de su modelo a partir de la tipología de Jesús visto como un segundo Moisés y el evangelio como una nueva Torah. Esta idea ya estaba expresada por Bacon en un trabajo anterior ${ }^{7}$. Tras esta tipología se esconde una agria discusión apologética. Bacon estima que el evangelio de Mateo es la gran refutación apostólica dirigida al judaísmo ${ }^{8}$. Esta propuesta de división en cinco libros, si bien acogida en la investigación posterior a Bacon, ha sufrido modificaciones y desarrollos. Por ejemplo, en los trabajos de Smith ${ }^{9}$, Allison y el de Green ${ }^{10}$. No sobra señalar que la propuesta de Bacon ha sido rebatida por investigadores mateanos tan importantes como Bornkamm y Kingsbury ${ }^{11}$.

Dada la gran cantidad de recursos estilísticos que Mateo despliega, se entiende que resulte realmente difícil proponer un modelo de su estructura literaria y teológica que gane la aceptación de las mayorías académicas. Asimismo, lo que explica esta historia de falta de consenso en la que se termina convirtiendo cualquier status quaestionis del tema es el gran riesgo que corre cualquier investigador de caer en cierto subjetivismo y unilateralismo a la hora de entender dichos recursos. De este peligro no está excluido este artículo. El objetivo que se propone aquí no es evaluar el status quaestionis, ni proponer otra alternativa de análisis. Formulado en positivo: en este artículo se pretende considerar si la propuesta de la estructura en cinco libros permite estudiar de manera controlada dichos recursos en los discursos del evangelio.

Siguiendo a U. Luz ${ }^{12}$, se parte de identificar ocho recursos literarios que ayudan a establecer la estructura de cada discurso. Estos son: a) agrupación de materiales similares en forma o contenido ${ }^{13}$; b) correspondencia lineal ${ }^{14}$; c) composición en arreglo a esquemas numéricos ${ }^{15}$; d) temas cen-

7 BACON, Sermon on the Mount, 46 y 106.

8 ID., "Five Books", 50.

9 SмIтн, "Fivefold Structure", 540-551.

10 Allison, New Moses; y Green, "Structure”, 7-59.

11 Bornkamm, “Bergpredigt”, 419-432; y Kingsbury, Matthew; ID., "Structure”, 451474.

12 Luz, Mateo. I, 37-42.

13 Por ejemplo, la serie de milagros de los capítulos 8-9, y la serie de parábolas de 21,28-22,14.

14 El primer y el último discursos son los más extensos (Mt 5-7 y Mt 24-25 respectivamente), mientras los discursos segundo y cuarto son los más cortos (Mt 10 y Mt 18 respectivamente).

15 Aunque los patrones numéricos binarios y septenarios tienen relevancia, entre otros, sin duda, el esquema más importante es el ternario. Por ejemplo, en 
trales sugeridos por la repetición de palabras clave ${ }^{16}$; e) versículos y textos centrales ${ }^{17}$; f) formación de dobletes ${ }^{18}$; g) inclusiones en conjuntos mayores y menores ${ }^{19} ; \mathrm{h}$ ) inclusiones circulares o quiasmos ${ }^{20}$. Citando a Luz, de la aplicación de los recursos literarios enumerados se pueden obtener los siguientes resultados:

El evangelio de Mateo se configura sobre todo en unidades menores. No es una serie de textos sueltos para fines litúrgicos o catequéticos. Su raíz vital es el estudio, la lectura, y esto desde el comienzo a la conclusión. Está escrito para una lectura reiterada. Se observa una cierta afinidad entre los recursos compositivos de Mateo y los recursos de la literatura veterotestamentaria y judía. Muchos de ellos tienen su contrapunto en el Antiguo Testamento; algunos resultan más comprensibles por la actividad escolar rabínica ${ }^{21}$.

tres secciones se distribuyen 1,18-2,23; 5,21-7,11 (5,21-32.33-48; 6,1-18.19-24; $7,1-11)$. Muchas veces son secciones principales y subsecciones compuestas con arreglo al número tres, por ejemplo $1,18-2,23$ y $2,13-23 ; 5,21-7,11 ; 5,21-33$ y $5,21-$ $26 ; 6,1-18 ; 6,7-15$ y 6,9-13 (2x3 peticiones), etc. Pero, como advierte el mismo Luz (Mateo. I, 38): "el número ternario es corriente en textos judíos. Hay que guardarse de interpretarlo a nivel de contenido, por ejemplo como número de la perfección. Se trata solo de un principio de sistematización literario y corriente en la instrucción oral".

${ }_{16}$ Por ejemplo, la frase ággelos kyríou ("el ángel del Señor") aparece 4 veces en 1,18-2,23; dikaiosýnē ("justicia") aparece 5 veces en 5-7; y apostéllō / apóstolos ("enviar/enviado") aparece 4 veces en 10,2-42.

${ }_{17}$ Como ejemplos de versículos centrales se pueden citar: 5,17.20.48; 6,1. 7.12.21; 10,16.26; 18,10.14. Ejemplos de textos centrales son: 5,17-20; 12,46-50; $22,34-40 ; 28,16-20$.

18 Las reduplicaciones crean un marco compositivo en torno a determinadas secciones (por ejemplo: 4,23/9,35; 19,30/20,16; 24,42/25,13 = inclusiones); descubren aspectos especialmente importantes (por ejemplo 9,13/12,7); y ayudan a tratar el mismo material bajo diversos aspectos (por ejemplo 10,17-22/24,9-13; 7,1619/12,33-35).

${ }_{19}$ Como ejemplo de inclusión en conjuntos mayores se puede presentar 1,24/28,20 (emmanuel/estaré con ustedes...). Ejemplo de inclusión en conjuntos menores es la frase final de 5,3.10, que define el contorno literario de las bienaventuranzas.

${ }^{20}$ En general, los quiasmos son una serie de inclusiones que envuelven un texto, con el esquema compositivo ABC / C'B'A'. Un ejemplo es la sección discursiva del Sermón del Monte (Mt 5-7), cuyo centro es el Padrenuestro. Fragmentos textuales menores con orden quiástico son, por ejemplo, 9,1b-8; 13,13-18; 18,10-14. Sobre los quiasmos en Mateo, véase FILson, "Broken Patterns", 227-231; y Lund, "Chiasmus", 405-433.

${ }^{21}$ Luz, Mateo. I, 42. 
En este artículo se van a aplicar dichos recursos en un análisis de las cinco secciones discursivas del evangelio. En el análisis también se siguen las sugerencias metodológicas propuestas por Wills y Keegan ${ }^{22}$.

\section{Aproximación y observaciones generales sobre la estructura y contenido de los discursos}

Se parte de la lectura del evangelio en las ediciones críticas del texto griego del Nuevo Testamento ${ }^{23}$. Según la opinión generalizada entre los comentaristas ${ }^{24}$, se puede percibir en las cinco unidades discursivas cierta simetría estructural, manifestada en: a) el primer y el último discurso son los más extensos ${ }^{25}$; aunque los tres discursos restantes constan de un capítulo cada uno, el segundo y cuarto discurso son menos extensos que el tercero $^{26}$. Temáticamente, el primer y el último discursos están conectados; asimismo, el segundo y el cuarto discursos. Sobre esto último, ya se había señalado que Mateo sugiere los temas principales de sus secciones mediante la repetición de palabras clave. En Mt $5-7^{27}$ no solo se sugiere la justicia del reino mediante la repetición de dikaiosýnē, sino también el tema de la fe, con los vocablos pístis / pisteuō (6 veces), que se repiten en 24-25 (6 ve430.

22 WILLS, "Scribal Methods", 241-257; KeEGAN, "Introductory Formulae", 415-

23 Aland, Novum Testamentum Graece, ad loc.; Holmes, Greek New Testament, ad loc.; O'Callaghan - Bover, Nuevo Testamento Trilingüe, ad loc.; Westcott - Hort, New Testament. I, ad loc.

${ }^{24}$ Además de los autores ya citados como defensores del modelo de los cinco libros, véase Osborne, Matthew, 40-47; MARK, "Literary Approaches", 51-52; France, Gospel of Matthew, 1-5; Viviano, “Mateo", 69-72; Luz, Mateo. I, 31-48; Bonnard, Mateo, 16-19; Schlatter, Matthäus; y Plummer, Commentary, xviii-xxv. En este último caso, seguimos la numeración original del texto, que diferencia el contenido de la introducción (en numeración romana) de aquella del comentario propiamente dicho (en numeración arábiga). Además de estos comentaristas, véase los análisis de ComBRINK, “Structure", 1-20; ID., "The Macrostructure", 61-90.

${ }^{25}$ El texto griego de Mt 5-7 consta de 1.988 palabras. Mientras Mt 24-25 consta de 1.575; con una diferencia de 413 palabras entre ambos. Todas las estadísticas de esta nota al pie de página, y la siguiente nota, son tomadas del software BibleWorks 4.0.

${ }^{26}$ Mt 10 consta de 719 palabras; mientras que Mt 18 consta de 667; con una diferencia de 52 palabras entre ambos. Por otro lado, Mt 13 consta de 1.072 palabras.

${ }^{27}$ En adelante, todas las citas realizadas solo con indicación de capítulo y versículo son referidas del evangelio de Mateo, salvo que se indique otra fuente. 
ces $)^{28}$. En el segundo discurso, el tema dominante es el envío, sugerido por la presencia de los vocablos apostéllō / apóstolos (4 veces); existe cierta afinidad con el tema del cuarto discurso, por cuanto son temas asociados a la vida comunitaria: asuntos relacionados con la disciplina en la comunidad, y manifiestos por la presencia de los vocablos adelfós (4 veces), mikrós (3 veces) y afiémi (4 veces).

$\mathrm{Si}$ estas tres indicaciones generales son correctas, se puede obtener un esquema concéntrico en la estructura de los discursos mateanos, una especie de gran sección discursiva de cinco libros (Mt 5-25). Dicha estructura concéntrica se puede expresar de la siguiente manera:

A 5-7: Sermón del Monte

B 10: discurso de envío

C 13: discurso parabólico

B' 18: discurso eclesial

A' 24-25: discurso escatológico

Interesa constatar la precisión tanto de las indicaciones generales antedichas, así como aquellas sobre la estructura concéntrica. Ahora se deben analizar más de cerca los inicios y los finales de estos discursos para verificar la exactitud en la delimitación de la esta hipótesis sobre la estructura concéntrica. Se comenzará con los finales de los discursos, por ofrecer indicios más claros de su intención redaccional.

\section{Las fórmulas finales de los discursos}

Al término del primer discurso se encuentra una frase de carácter conclusivo: kaì egéneto hóte etélesen ho Iēsoûs toûs lógous toútous... (“"y aconteció que cuando terminó Jesús estas palabras...”; Mt 7,28). Frases similares a esta se repiten al final de los otros cuatro discursos:

11,1: kaì egéneto hóte etélesen ho Iēsoûs diatássōn toîs dốdeka mathētaîs autoû...

13,53: kaì egéneto hóte etélesen ho Iēsoûs tàs parabolàs taútas...

19,1: kaì egéneto hóte etélesen ho Iēsoûs toûs lógous toútous...

26,1: kaì egéneto hóte etélesen ho Iēsoûs pántas toûs logous toútous...

28 Así lo sugiere LoHR, “Oral Techniques”, 428-429. 
Esta repetición de frases tan similares al final de los cinco discursos ha hecho pensar a algunos comentaristas que dichas frases son un recurso estilístico del evangelista, o de su tradición (¿fuente especial mateana, $Q$ ?) ${ }^{29}$. El análisis de esta fórmula de finalización permite elaborar los siguientes comentarios.

En primer lugar, la fórmula concreta se compone de la frase kaì egéneto hóte + verbo teléo + ho Iēsoûs como sujeto. Se ha sugerido que la fórmula no proviene de la redacción mateana, sino de su tradición ${ }^{30}$. De hecho, el vocabulario de la fórmula es impropio al estilo redaccional mateano. Egéneto hóte no es mateano ${ }^{31}$; aunque el verbo teléo proviene de la redacción mateana ${ }^{32}$, aparece dos veces en la tradición (v.g.: 10,23; 17,24) ${ }^{33}$; la indicación de los discursos con el sustantivo lógoi proviene de $Q$ (cf.

29 Por citar solo algunos, están: Davies, Matthew, 71; Luz, Mateo. I, 582-583; ZAHN, Matthäus, 324; Allen, Commentary, 64; Bacon, Sermon on the Mount, 167-168. Véase también a ZerWick - Grosvenor, Análisis gramatical, 25; BultMAnn, Tradición sinóptica, 394; Moulton, Grammar, 41; Stendahl, School, 25. Sumario de opiniones en: Rolg CerVERA, Estructura, 6.

30 Luz, Mateo. I, 582; HawkIns, Horae Synopticae, 132-133.

31 La construcción gramatical kaí egéneto + verbo finito en indicativo es considerado como un septuagintismo, es decir, un fenómeno de traducción de la construcción hebrea: $w^{e}$ yehî + verbo finito en indicativo y complemento. Como ejemplo, véase Gn 6,1: “y aconteció (TM: weyehî; LXX: kai egéneto) que comenzando los hombres a multiplicarse sobre la faz de la tierra". Al parecer, el traductor griego entendió la relación sintáctica de la primera parte de la oración hebrea de forma diferente. La coordinación existente entre las frases kî-ḥehel l Hifîl + kîl) y lārôb $(Q a l+l$, construcción de infinitivol la entendió como independientes y, por ello, las traduce como hēníka ếrxanto y polloi gínesthai, pero respetando el perfecto de la primera frase, que lo traduce por el aoristo indicativo y el infinitivo de la última. No obstante esta situación, la estructura sintáctica está presente: kaí egéneto + verbo finito en indicativo y complemento. Sobre esto, véase MuraokA, Greek-Hebrew-Aramaic, 24; ID., Greek English-Lexicon, 131; Zerwick, Griego del Nuevo Testamento, § 389 (nota 2); y ReILING, “Kai Egeneto", 153-163. Delitzsch (Hebrew New Testament) retrotraduce al hebreo la frase griega kai egéneto hóte etélesen ho lēsoûs como wayhî kekallôt Yēšû'a en Mt 11,$1 ; 13,53 ; 19,1$ y 26,1 .

32 El verbo teléō aparece siete veces en Mt, ninguna vez en Mc y cuatro veces en Lc. El uso del aoristo etélesen solo se da en Mt en las cinco fórmulas finales. Nótese también que epeidé (en Lc 7,1) es un hapax legomena, usado en sentido temporal (su uso más común es causal). Sobre esto, véase Blass - Debrunner - Funk, A Greek Grammar, § 445.1.

33 Luz (Mateo. II, 151) no está seguro sobre la proveniencia de Mt 10,23, y señala tanto la tradición especial mateana $(M)$, como una edición particular de $Q\left(Q^{\mathrm{Mt}}\right)$. El caso de Mt 17,24 es único. No se puede tener seguridad de su fuente y lo más probable es que el v. 24, así como todo el pasaje (v.g.: vv. 24-27), provenga de M. La razón para pensar así consiste en que el verbo teléō con el sentido de “cumplir con 
Lc 6,47). Sobre esto último se debe notar, aunque con cautela, que en Ex 20,1 (LXX) se llama al decálogo lógoi hoûtoi. No obstante, se debe recordar que es la tradición mateana, y no Mateo mismo, quien emplea este vocabulario. Por otro lado, los paralelos de la LXX en Dt $(31,1.24 ; 32,44-$ 46; o Nm 16,31; Jr 33,8) no dejan de ser simplemente sugestivos, pero poco argumento aportan al tema del paralelismo entre Jesús y Moisés, o entre el Sermón del Monte y el Decálogo. En esta línea, resultan más instructivos y mejor fundamentados como paralelos Ex 19 (a Mt 5,1) y Ex 34,29 (a Mt 8,1).

Mt 5,1: ....anébē eis tò óros...

Ex 19,3: kaî Mōusēs anébe eis tò óros tô̂ Theoû...

Mt 8,1: katabántos dè autoû apò tô̂ órous...

Ex 34,29: hōs dè katébainen Mōusēs ek toû órous...

Al parecer, estas referencias procederían del trabajo redaccional mateano. Así lo sugiere el carácter redaccional de la frase idōn de +ójloi (véase 8,$18 ; 9,36$ ) y la presencia de léxico redaccional como oros y ójloi ${ }^{34}$. En este sentido, se podría decir que, si bien la tradición mateana vería una relación entre el sermón de Jesús y el decálogo veterotestamentario, para Mateo el punto de contraste se daría entre las figuras de Moisés y Jesús, sus respectivos escenarios y los colectivos convocados en torno a sus códigos.

En segundo lugar, si se observa con cuidado el paralelo entre Mt 7,28 y Lc 7,1, se podrá notar que, aunque cambia la redacción concreta en cada uno, según los gustos estilísticos y las líneas teológicas de cada evangelista, la construcción gramatical es esencialmente la misma ${ }^{35}$.

Mt 7,28: kaì egéneto hóte etélesen ho Iēsoûs toûs lógous toútous...

Lc 7,1: epeidé eplếrōsen pánta tà rhếmata autoû...

Esto hace suponer la presencia de una construcción gramatical $Q$ tras ambas redacciones, aunque ya no sea posible reconstruirla ${ }^{36}$.

los impuestos" no ocurre nunca más en todo el NT: un hapax legomena, como lo llama Luz (Mateo. II, 689, n. 4).

34 Sobre esto, véase Luz, Mateo. I, 68, 275 (nota 2); Mateo. II, 28 (nota 1); y BultMANN, Tradición sinóptica, 393.

${ }^{35}$ Luz (Mateo. I, 582) nota que Mateo bien pudo cambiar el verbo plēróō de $Q$ por el verbo teléō a causa del eco cristológico que tiene este vocablo en su teología.

36 La indeterminación sobre este asunto viene expresada en la redacción $Q 7,1$ así: [[kai egéneto]] e[[plếrō]]sen... toùs lógous toútous... La razón para cerrar entre 
En tercer lugar, también se debe notar que en tres de los discursos la fórmula kaì egéneto hóte está ligada a un verbo de movimiento (cf. $11,1 ; 13,53 ; 19,1)^{37}$. Asimismo, en dos de los discursos la fórmula está conectada expresamente a las multitudes (ójloi; cf. 7,28; 19,2) ) $^{38}$. También en dos de los discursos, la fórmula está dirigida expresamente a los discípulos (cf. 11,1; 26,1). Solo en dos de los discursos, a la fórmula kaì egéneto hóte le sigue un comentario redaccional (cf. 7,29; 19,2). En particular, es notorio el origen tradicional del comentario y el trabajo redaccional en 7,28b-29.

7,28 Y aconteció que, cuando Jesús terminó estas palabras, la multitud se asombraba por su enseñanza (kaì egéneto hóte etélesen ho Iēsoûs toûs lógous toútous, exeplếssonto hoi ójloi epì tē didajē autoû), 29 porque estaba enseñándoles (ēn gàr didaskōn autoùs) como quien tiene autoridad y no como sus escribas ${ }^{39}$.

doble corchete el verbo plēróō (dejando por fuera la "forma” del aoristo), es la duda entre los verbos, mateano (teléō) y lucano (plēróō). Cf. Robinson - Hoffmann - KLopPENBORG, Documento Q, 122. Los dobles corchetes califican con C, en escala de A-U, el grado de indeterminación de los editores. Esta misma reconstrucción y valoración aparece en HofFMANN - HEIL, Spruchquelle Q, 46.

37 Mateo utiliza dos veces el verbo metaírō (en 13,53 y 19,1); la tercera vez emplea el verbo metabaínō (en 11,1). Los tres verbos aparecen en indicativo de aoristo de la tercera persona del singular, tal como lo exige el contexto narrativo de cada escena.

38 Keegan ("Introductory Formulae”, 425-428) sugiere que existe una diferencia considerable en el uso mateano entre el singular (ójlos) y el plural (ójloil). Para este autor, el plural indica un uso técnico (v.g.: 4,25; 5,$1 ; 9,3 ; 13,12 a ; 13,36)$. En este artículo se adopta la tesis general de Keegan, pero sin pretender extraer las conclusiones que han extraído sobre este tema Cousland, Crowds; CARTER, "Crowds", 5467; MineAR, “Disciples”, 28-44; y VAN TILBORG, Jewish Leaders, 164, 171, quienes, en general, consideran que las ójloi son un personaje corporativo, positivo, que no solo acepta a Jesús, sino que incluso lo sigue. Van Tilborg llega a considerar a las ójloi como una retroproyección teológica de la experiencia de discipulado de la comunidad mateana. Estas tesis son contrarias a las de Kingsbury (Matthew as Story, 2425), quien, en el otro extremo, tiende a concebir de una forma muy negativa a las ójloi. Más adelante se tendrá ocasión de analizar la tesis de Keegan. Por ahora se dejan anotadas estas explicaciones generales.

39 Debido a la importancia del pasaje para la discusión que se sigue, se ofrece una traducción castellana. La traducción es propia. Se anotan dos observaciones de traducción. En la frase epì tē didajē autoû el adverbio epí seguido por frase de dativo indica la causa de la emoción; por ello la traducción "por su enseñanza”. Sobre esto, Zerwick, Griego del Nuevo Testamento, § 126. Asimismo, en la frase ēn gàr didaskōn autoùs se da el caso del uso de la construcción perifrástica por medio del uso del verbo eînai + participio en lugar de la forma simple del verbo l"estaba en- 
El carácter redaccional viene muy marcado en 7,29, por dos razones: a) la partícula gár subraya la función explicativa del versículo; y b) la presencia del versículo proviene de Mc 1,22 (nótese que parte del vocabulario del v. 28 también proviene de Mc 1,22). Solo se señala en negrita el trabajo redaccional mateano:

Mt 7,28b-29: exeplếssonto hoi ójloi epì tē didajē autoû, ēn gàr didaskōn autoùs hōs ejousían éjōn, kaì ouj hōs hoi grammateîs autōn.

Mc 1,22: exeplếssonto epì tē didajē autoû, èn gàr didaskōn autous hōs ejousían éjōn, kaì ouj hōs hoi grammateîs.

Todo esto sugiere que Mateo desplazó el lugar de la enseñanza paradigmática en Cafarnaúm de su lugar tradicional, y la reemplazó con su primer discurso: el Sermón del Monte.

Finalmente, en el último discurso de Mt 26,1, la fórmula kaí egéneto hóte está marcada con el adjetivo pâs para expresar la finalización de la sección de los cinco grandes discursos y el inicio de la última sección del evangelio: el relato de la Pasión/Resurrección (Mt 26-28).

También se debe concluir que en estas observaciones sobre la fórmula mateana se encontró un patrón estereotipado con el cual Mateo señala el término de la sección discursiva. Dicha fórmula final de los discursos no es redaccional, sino que asume los usos de la tradición, tanto en vocabulario como en expresión. De hecho, la fórmula en sí sugiere un uso griego semitizante, a la manera de los septuagintismos, pero con un estilo propio (cf. el uso del kaí egéneto + hóte, muy diferente al lucano, por ejemplo). Es fácil sospechar del trabajo redaccional de algún tipo de escuela de escribas cristianos. Pero sobre este problema se debería adelantar un análisis más profundo de la estructura sociológica y la teología de Mateo, y no solo en el estilo redaccional del evangelio. Por el espacio con el que se dispone y el tema que se sigue, aquí solo se pueden compartir las intuiciones de Wills ${ }^{40}$.

Por otro lado, el resultado más importante del análisis fue precisar con exactitud el final de estos cinco discursos mateanos:

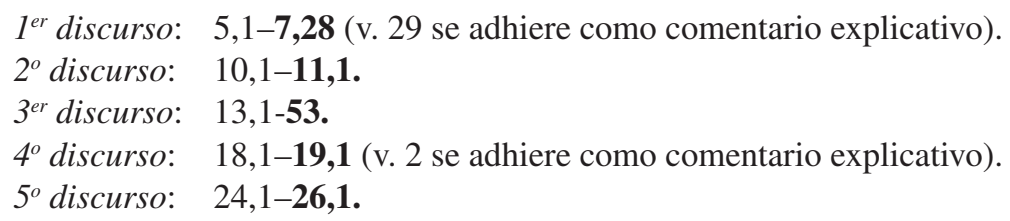

señando" = "enseñaba"). Sobre esto, véase también a ZERWICK, Griego del Nuevo Testamento, $\S 360$.

${ }^{40}$ WILLS, "Scribal Methods", 241-257. 
Es momento de dirigir el análisis al inicio de los discursos, buscando patrones e indicios que permitan establecer un uso redaccional mateano.

\section{El inicio de los discursos ${ }^{41}$}

En contraste con los finales de los discursos, al inicio no se encuentra ninguna frase que sugiera una fórmula fija. No obstante, se observan ciertos patrones y recursos de estilo que se concretan en el uso reiterativo de palabras clave asociados al motivo de los discursos. Se presentan los textos, señalando en negrita las palabras y frases clave.

\section{5,1: tous ójlous... kaì kathísantos autoû prosēlthan autō hoi mathētai} autô̂.

10,1: kaì proskalesámenos toùs dốdeka mathētas autoû...

13,1-2: en tē hèmera ekeinē exelthốn ho Iēsoûs tès oikías ekáthēto parà tēn thálassan, kaì synếjthēsan pròs autòn ójloi polloì... kathēsthai...

18,1: en ekeinē hốra prosēlthon hoi mathètaì tō Iēsoûs legontes...

24,1.3: Kaì exelthốn ho Iēsoûs apò toû hieroû eporeúeto, kaì prosēlthon hoi mathētaì autô̂ epidê̂xai autō tàs oikodomàs toû hieroû... Kathēménou dè autoû epì toû órous tōn elaiōn prosēlthon autō hoi mathètaì kat' idían légontes...

Se observa la reiterada presencia del verbo kathízo ${ }^{42}$, acompañado del verbo prosérjomai o légō; y los sustantivos que designan a las ójloi; y a

${ }^{41}$ Se siguen las sugerencias metodológicas de KeEGAN, “Introductory Formulae”, 415-430.

42 La presencia de este verbo es significativa: refleja la costumbre judía de enseñanza. Aunque esta costumbre se extendía por todo el cercano Oriente antiguo (CIVIL, “Education in Mesopotamia”, 303), al parecer, la postura sedente en la instrucción es relativamente reciente en el judaísmo. Así lo sugiere el tratado del Talmud, Meg 21a: “los rabinos enseñaban: desde los días de Moisés hasta los días del rabino Gamaliel (I, el maestro del apóstol Pablo) la Torá se estudiaba de pie. Pero desde que el rabino Gamaliel murió, la enfermedad cayó sobre el mundo, y se aprende la Torá sentado". Véase lo que dice la Mishna, Meg 4, 1: "el que lee el rollo puede estar en pie o estar sentado. Si lo lee uno o si lo leen dos, cumplen la obligación". Si bien el texto talmúdico está en concordancia con otros datos neotestamentarios, como Lc 4,16.20 (donde se explicita que Jesús se levanta para leer y, luego, se sienta para comentar el pasaje isaiano leído), y Hch 22,3. ABERBACH ("Change", 173-174), que ha estudiado el pasaje talmúdico en mención, sostiene que se debe considerar que el cambio en la postura de la instrucción se debió dar durante el siglo । d. C., como parece indicar el pasaje. El texto misnaico es tomado de DEL VALLE (La Misná, 307). El texto talmúdico es traducción propia, tomado de la traduc- 
los mathètaí. La presencia conjunta de estos vocablos en solo tres de los cinco textos $(5,1 ; 13,1-2 ; 18,1)$ sugiere un análisis más detenido en el contexto para buscar mayor precisión.

\subsection{Observaciones sobre Mt 5,1}

Como señala Luz en su comentario ${ }^{43}$, Mt 5,1 recuerda el sumario inmediatamente anterior de 4,23-25. La función de este sumario consiste en adelantar no solo la sección discursiva (Mt 5-7), sino también la sección narrativa (Mt 8-9) o, tomando prestada la elocuencia de Schniewind: "el Mesías de la palabra, el predicador, es descrito en los capítulos 5-7, y el Mesías de la obra, el sanador, en los capítulos 8-9"44.

Resulta significativo que este primer sumario (Mt 4,23-25), así como aquel segundo de Mt 9,35-38, anticipe la sección discursiva y esté conectada con ella por la presencia del vocablo ójloi. Se podría decir que, aunque el inicio expreso del discurso aparece en 5,1, este ya viene siendo introducido desde 4,25.

\subsection{Observaciones sobre Mt 10,1}

El discurso realmente solo inicia en el v. 5; en los vv. 1-4 aparece la lista de los nombres de los Doce. Pero en estos cinco versículos no se encuentra ninguno de los vocablos vistos al inicio de los otros discursos.

La última perícopa de Mt 9 (vv. 35-38) es un sumario de la predicación y acción de Jesús, donde también aparece el vocablo ójloi (v. 36). Desde un punto de vista narrativo, en él se une y da sentido a la lista de los Doce que le sigue y al discurso de envío. El texto de importancia para los fines de este estudio está en los vv. 36-38:

${ }^{36}$ Y viendo a las multitudes (toùs ójlous) se conmovió profundamente por ellos (esplagjnísthē peri autōn), porque estaban afligidos e indefensos (hóti hēsan

ción alemana ofrecida por STRACK - BILLERBECK (Kommentar, 763) y que dado su interés aquí reproducimos: "die Rabbanan haben gelehrt: seit den Tagen Moses bis zu Rabban Gamaliël (I., dem Lehrer des Apostels Paulus) hat man die Tora nur stehend gelernt; seitdem aber Rabban Gamaliël tot ist, kommt Krankheit herab auf die Welt und lernt man die Tora sitzend".

${ }^{43}$ Luz, Mateo. I, 49-250.
44 SchnieWInd, Matthäus, 36. 
eskylménoi kai ettimménoil como ovejas que no tienen pastor. ${ }^{37}$ Entonces les dijo a sus discípulos: "aunque la mies es mucha, sin embargo, los trabajadores pocos (ho mèn therismòs polýs, hoì dè ergátai holígoi); ${ }^{38}$ pidan pues al señor de la mies para que envié trabajadores a su mies" 45 .

La necesidad de enviar discípulos se expresa con la doble imagen del pueblo visto como rebaño/mies, que no tiene pastores/obreros. La frase sobre la petición elevada a Dios (v. 38) recibe su desarrollo tanto en el listado de los Doce (vv. 1-4) como en el discurso de envío: ellos son los obreros enviados a la mies. Se puede decir que, desde un punto de vista narrativo, el discurso de envío comienza en Mt 9,36.

Como nota Keegan ${ }^{46}$, la presencia del vocablo ójloi resulta muy significativo, ya que su presencia en otros lugares también señala un punto de quiebre en la narración; sobre todo en 4,25 y 13,36. ¿Se podría precisar algo más sobre estos inicios de discursos en estos dos últimos pasajes, a saber 4,25 y 13,36 ? Al menos, con lo ya visto al comentar sobre $4,25-5,1$, se podría esperar que en 13,36 también se presente un cambio de escenario y personajes. Una pista para la solución de este planteamiento parece darse si se lee el inicio de Mt 13 con relación a 13,36.

\subsection{Observaciones sobre Mt 13,1-2.36}

13,1 En aquel día, saliendo Jesús de su casa se sentó a la orilla del mar. 2 Y se congregaron junto a él tantas multitudes que, subiéndose en una embarcación, se sentó (kaì synếjthēsan pròs autòn ójloi polloú, hốste autòn eis ploîon embánta kathēsthai), y toda la multitud permanecía en la playa ${ }^{47}$.

45 Debido a la importancia del pasaje para la discusión que se sigue, se ofrece una traducción castellana. La traducción es propia. Se anotan varias observaciones de traducción. El aoristo esplagjnísthē indica el conmoverse con las partes internas o profundas. El participio eskylménoi viene del verbo skýllō ("despellejar, afligir, acosar") y el participio ettimménoi ("desamparados, indefensos"), ya que proviene del verbo rhíptō ("arrojar, echar"). Finalmente, en la frase ho mèn therismòs polýs, hoi dè ergátai holígoi, la construcción mèn... dè es equivalente a la latina quidem... autem a la cual ZERWICK - GROSVENOR (Análisis gramatical, 33) ofrecen tres opciones de traducción: al "por un lado... por el otro; b) “... (efectivamente)... pero”; y c) "aunque... sin embargo".

46 KeEgan, “Introductory Formulae”, 427-428.

47 La traducción es propia. En el v. 2, la construcción gramatical hóste con acusativo + infinitivo sugiere un sentido consecutivo o de resultado. Es decir, Jesús no tuvo más opción que sentarse en una barca a causa de la multitud que colmaba la orilla. Sobre esto, véase Zerwick - Grosvenor, Análisis gramatical, 47. 
13,36 Entonces, dejando a las multitudes (toùs ójlous), entró en la casa. Y acercándosele sus discípulos (kaì prosēlthon autō hoi mathētai autoû), le decían: "explícanos (diasáfēson hēmîn) la parábola de la cizaña del campo" 48 .

Como señala Keegan ${ }^{49}$, el cambio de lugar y de audiencia ${ }^{50}$ muestra un cambio paradigmático en el evangelio. Este cambio se observa en que, desde 13,36, todos los discursos son dirigidos exclusivamente a sus discípulos. Nótese que los discursos de 5-7; 10 y 13 estaban dirigidos tanto a sus discípulos como a las multitudes. A partir de 13,36, el discurso del capítulo 18 y el de los capítulos 24-25 estarán dirigidos exclusivamente a sus discípulos.

\subsection{Observaciones sobre Mt 24,1-3}

En este pasaje se percibe el cambio que se da entre los vv. 1-2 y el v. $3^{51}$.

24,1 Y saliendo Jesús del templo, se iba (kaì exelthốn ho Iēsoûs apò toû hieroû eporeúeto); y se acercaron sus discípulos para mostrarle las construcciones del templo (epideîxai autō tàs oikodomàs toû hieroû). $2 \mathrm{Y}$ él les contesta diciendo (ho dè apokritheìs eîpen autô̂s): "¿no ven todo esto? De cierto les digo que no quedará piedra sobre piedra que no sea demolida" 52 .

${ }^{48}$ La traducción es propia. Diasáfeō es un verbo compuesto (diá + sáfeō) que se deriva del sustantivo safēs ("claro"), y significa: ex-plicar, como lo han indicado ZerWick - Grosvenor, Análisis gramatical, 52.

49 KeEgan, “Introductory Formulae”, 427-428.

50 El desplazamiento espacial está indicado en Mt 13,36 con la frase êlthen eis tến oikían ("entró en la casa"; cf. Mt 13,1). El cambio de personajes del versículo explica que se dejan a los personajes que venían desde Mt 13,2 (las ójloi), y que su nueva audiencia son ahora los mathētaí.

51 La mayoría de los comentaristas lo ha notado. Davies (Matthew, 190) señala el v. 3 como inicio del discurso. Asimismo: Luz, Mateo. III, 499-500; Bultmann, Tradición sinóptica, 96; Zahn, Matthäus, 662-663; Plummer, Commentary, 329; Allen, Commentary, 254.

52 La traducción es propia. Observe en la frase kai exelthón ho lēsoûs apò toû hieroû el uso de apó en lugar de ek. Nótese también en la misma frase (kai exelthốn ho lēsoûs apò toû hieroû eporeúetol el uso del imperfecto indicativo eporeúeto, enunciando que Jesús sigue caminado mientras estaba saliendo (exelthốn). 
La ubicación espacial y la secuencia temporal, con referencia a la perícopa anterior al pasaje, vienen dadas por la frase participial: kaì exelthốn ho Iēsoûs apò toû hieroû eporeúeto. Inmediatamente, sigue el inicio del diálogo entre Jesús y sus discípulos con el verbo epideíkdymi ("señalar, mostrar"), que expresa una "pregunta" implícita en el gesto mismo, y la respuesta explícita de Jesús encabezada con el verbo apokrínomai. Estos datos son suficientes para señalar el comienzo de un discurso. Pero, después de estos claros indicios, en el v. 3 se ofrecen nuevos datos que sugieren el inicio de un nuevo diálogo, dando la impresión de que el actual 24,1-3 es fruto de la reelaboración de más de un material tradicional sobre el discurso escatológico.

24,3 Y sentado en el monte de los Olivos se le acercaron los discípulos en privado diciendo (hoi mathêtaì kat' idían légontes): "dinos: ¿cuándo será esto, y cuál la señal de tu parusía y fin del mundo?" ${ }_{53}$

Si se toma nota de los lugares paralelos (Mc 13 y Lc 21), se observará que el discurso apocalíptico (Mc 13|Mt 24-25|Lc 21) es iniciado en cada evangelio con el apotegma sobre la destrucción del templo: blépeis taútas tàs megálas oikodomàs, ou mế afethē hōde lithos epì lithon hòs ou $m \bar{e}$ katalythē (Mc 13,2IMt 24,2ILc 21,6) ${ }^{54}$. También se notará que este apotegma es transformado redaccionalmente por cada evangelista. En especial, el encuadre narrativo del apotegma (i.e. Mc 13,1IMt 24,1।Lc 21,5). Finalmente, el hecho de que cada evangelista utiliza este apotegma como introducción al discurso apocalíptico se nota en el desarrollo en su transformación redaccional, siendo Lucas el caso más notorio. Ya en Lc 21,7 se convierte en simple indicación de explicación lo que en Mt 24,3 (y Mc 12,3) es una segunda ubicación del marco espacial ${ }^{55}$.

Todo esto lleva a sospechar que en Mt 24,1.3 se presentan dos comienzos diferentes de la tradición mateana al discurso apocalíptico unidos redaccionalmente. Es decir, aunque en el estado actual de la narración el inicio del discurso se da en Mt 24,1-3, se debe precisar diciendo que el ini-

${ }^{53}$ La traducción es propia. En la frase kat' idían, se debe tener en cuenta que el vocablo ídios implica aquello propio y, por ende, privativo o separado.

54 Lc 21,6 evita la frase inicial de Mc | Mt. El primer evangelio parece seguir una redacción, o tradición diferente de la redacción actual marcana ¿¿de la tradición especial mateana? en $Q$ no aparece el discurso escatológico).

55 Nótese que en Lc 21,7 se omite toda la frase de Mc 13,3|Mt 24,3. Podría ser que Lucas ve en la simple acción de preguntar de los discípulos, toda la serie de acciones descrita en Mc 13,3. Por otro lado, Mt 24,3 modifica redaccionalmente toda la frase. 
cio del discurso se da propiamente en Mt 24,3, siendo Mt 24,1-2 un encuadramiento previo. En todo caso, es en Mt 24,3 donde se observa la presencia de los verbos kathízō, prosérjomai y légō; y los sustantivos plurales ójloi y mathètaí.

Al finalizar esta sección de los inicios de los discursos se puede concluir, junto con Keegan ${ }^{56}$, diciendo que Mateo usa una terminología específica para iniciar sus discursos, precisamente con el uso intencional y regular de los verbos kathízō, prosérjomai, légō y los sustantivos ójloi y mathētaí.

\section{Conclusión}

En este análisis de las secciones narrativas se ha podido precisar no solo la fórmula empleada por Mateo para finalizar sus discursos, sino también los recursos léxicos y narrativos usados al inicio de las nombradas secciones. Mientras la fórmula final de los discursos es: kaì egéneto hóte + verbo teléo + ho Ièsoûs como sujeto, los recursos léxicos son tres: a) el uso de los siguientes verbos: kathízo, prosérjomai y légō; b) el empleo de los siguientes sustantivos: ójloi y mathētaí; y c) en algunos casos (primer y segundo discursos), la presencia anticipadora de sumarios (cf. 4,23-25; 9,35-38).

Finalizamos el análisis ofreciendo un elenco de la ubicación exacta del inicio y el final de las cinco secciones discursivas mateanas.

\section{Discurso y temática}

$1^{\text {er }}$ discurso: sobre la justicia en el reino

$2^{\circ}$ discurso: de envío

$3^{\text {er }}$ discurso: sobre el reino de los cielos, en parábolas

$4^{\circ}$ discurso: eclesiástico

$5^{\circ}$ discurso: escatológico

\author{
inicio-final \\ 4,25-7,28 \\ $9,35-11,1$ \\ $13,1-[13,36]^{57} 13,53$ \\ $18,1-19,1$ \\ $24,3-26,1$
}

56 KeEgan, “Introductory Formulae”, 428.

57 Se recuerda el giro paradigmático que 13,36 da entre la enseñanza pública, dirigida a las multitudes, de los dos primeros discursos ly la primera parte del tercero), y la enseñanza privada de los dos últimos discursos, dirigida al grupo de los discípulos más cercanos. 


\section{Bibliografía}

Aberbach, M., "The Change from a Standing to a Sitting Posture by Students after the Death of Rabban Gamaliel", JQR 52 (1961) 168-174

Aland, B. y K. et al. (eds.), Novum Testamentum Graece, Stuttgart ${ }^{28} 2012$.

Allen, W. C., Critical and Exegetical Commentary on the Gospel According to St. Matthew (International Critical Commentary), New York 1907.

Aluison, D. C., Jr., The New Moses: A Matthean Typology. Minneapolis 1993.

-, "The Structure of the Sermon on the Mount", JBL 106 (1987) 423-445.

BACON, B. W., Studies in Matthew. New York 1930.

-, "Jesus and the Law: A Study of the First Book of Matthew (Mt. 3-7)": JBL 47 (1928) 203-231.

-, "The 'Five Books' of Matthew against the Jews", Exp 8/15 (1918) 56-66.

-, The Sermon on the Mount: Its Literary Structure and Didactic Purpose, New York 1902.

BibleWorks 4.0 - The Premier Biblical Exegesis and Research Program CD-ROM, Montana 1999.

Blass, F. - Debrunner, A. - Funk, R., A Greek Grammar of the New Testament and other Early Christian Literature, Chicago 1961.

Bornkamm, G., "Der Aufbau der Bergpredigt", NTS 24 (1978) 419-432.

Bonnard, P., Evangelio según san Mateo, Madrid 1976.

Bultmann, R., Historia de la Tradición sinóptica (Biblioteca de Estudios Bíblicos 102), Salamanca 2000.

Carter, W., "The Crowds in Matthew's Gospel”, CBQ 55 (1993) 54-67.

Civil, M., "Education in Mesopotamia", en D. N. FreEdman (ed.), Anchor Bible Dictionary. II. Garden City, NY, 1992, 301-305.

Combrink, H. J. B., "The Structure of the Gospel of Matthew as Narrative", TynBul 34 (1983) 61-90.

-, "The Macrostructure of the Gospel of Matthew", Neot 16 (1982) 1-20.

Cope, O. L., Matthew: A Scribe Trained for the Kingdom of Heaven (Catholic Biblical Quarterly Monograph Series 5), Washington 1976.

Cousland, J. R. C., The Crowds in the Gospel of Matthew (Novum Testamentum Supplements Series 102), Leiden 2002.

DAVIES, M., Matthew (Readings: A New Biblical Commentary), Sheffield 2009.

Delitzsch, F., Neue Untersuchungen über Entstehung und Anlage der kanonischen Evangelien. I, Leipzig 1853.

-, Hebrew New Testament, Berlin 1901.

Eldis, P. F., Matthew: His Mind and his Message, Collegeville 1974. 
Fenton, J., "Inclusio and Chiasmus in Matthew”, en E. A. Livingstone (ed.), Studia Evangelica. I (Texte und Untersuchungen zur Geschichte der Altchristlichen Literaur 73), Berlin 1959, 174-179.

Filson, F. V., "Broken Patterns in the Gospel of Matthew", JBL 75 (1956) 227-231.

France, R. T., The Gospel of Matthew (The New International Commentary on the New Testament), Grand Rapids 2007.

Green, H. B., "The Structure of Matthew's Gospel”, en E. A. Livingstone (ed.), Studia Evangelica. IV (Texte und Untersuchungen zur Geschichte der Altchristlichen Literaur 102), Berlin 1968, 47-59.

Hawkins, J. C., Horae Synopticae: Contributions to the Study of the Synoptic Problem, Oxford 1899.

Hoffmann, P. - Heil, C. (eds.), Die Spruchquelle Q: Studienausgaben, Leuven 2002.

Holmes, M. W. (ed.), The Greek New Testament: Society of Biblical Literature Edition, Atlanta 2010.

KeEgAn, T. J., "Introductory Formulae for Matthean Discourses", CBQ 44 (1982) 415-430.

Kingsbury, J. D., Matthew as Story, Philadelphia 1988.

-, Matthew, Structure, Christology, Kingdom, Philadelphia 1975.

-, "The Structure of Matthew's Gospel and his Concept of Salvation", $C B Q$ 35 (1973) 451-474.

LoHr, C. H., "Oral Techniques in the Gospel of Matthew", CBQ 26 (1961) 427-430.

Lund, N. W., "The Influence of Chiasmus upon the Structure of the Gospel according Matthew", AThR 13 (1931) 405-433.

Luz, U., El Evangelio según san Mateo. IV (Biblioteca de Estudios Bíblicos 115), Salamanca 2003.

-, El Evangelio según san Mateo. III (Biblioteca de Estudios Bíblicos 111), Salamanca 2003.

-, El Evangelio según san Mateo. II (Biblioteca de Estudios Bíblicos 1030), Salamanca 2001.

-, El Evangelio según san Mateo. I (Biblioteca de Estudios Bíblicos 74), Salamanca 1993.

Mark, A. P., "Literary Approaches and the Gospel of Matthew", en M. A. Powell (ed.), Methods for Matthew, Cambridge 2009, 44-82.

Minear, P. S., "The Disciples and the Crowds in the Gospel of Matthew", AThR Supplement 3 (1974) 28-44.

Moulton, J. H., Grammar of New Testament Greek. IV, Edinburgh 1976. 
Muraoka, T., A Greek-Hebrew-Aramaic: Two Way Index to the Septuagint, Louvain 2010.

-, A Greek English-Lexicon of the Septuagint, Louvain 2009.

NEIRYNCK, F., "La rédaction matthéenne et la structure du premier Évangile", en I. DE la Potterie (ed.), De Jésus aux Évangiles: tradition et rédaction dans les Évangiles synoptiques (Bibliotheca Ephemeridum Theologicarum Lovaniensium 25), Gembloux 1967, 41-73.

O'Callaghan, J. - Bover, J. M. (eds.), Nuevo Testamento Trilingüe (Biblioteca de Autores Católicos 100), Madrid ${ }^{4} 1999$.

Osborne, G. R., Matthew (Baker Exegetical Commentary on the New Testament 1), Grand Rapids 2010.

Plummer, A., An Exegetical Commentary on the Gospel According to St. Matthew (International Critical Commentary), New York 1910.

ReILING, J., "The Use and Translation of Kai Egeneto, 'And it Happened', in the New Testament", BT 16 (1965) 153-163.

Robinson, J. M. - Hoffmann, P. - Kloppenborg, J. S. (eds.), El Documento $Q$ en Griego y en Español (Biblioteca de Estudios Bíblicos 107), Salamanca 2002.

Roig Cervera, M. A., La Estructura Literaria del Evangelio de San Mateo (Tesis Doctoral Presentada a la Facultad de Filología; Dpto. de Filología Griega e Indoeuropea), Madrid 1995.

Schlatter, A., Von, Die Kirche des Matthäus (Beiträge zur Förderung christlicher Theologie 33/1), Gütersloh: Bertelsmann 1929.

Schniewind, J. Das Evangelium nach Matthäus (Das Neue Testament Deutsch 21), Göttingen ${ }^{8} 1968$.

Sмith, C. R., "Literary Evidences of a Fivefold Structure in the Gospel of Matthew", NTS 43 (1997) 540-551.

Stanton, G., "The Origin and Purpose of Matthew's Gospel Matthean Scholarship from 1945 to 1980", en ANRW II-25/3, 1890-1951.

Stendahl, K., The School of St. Matthew: and Its Use of the Old Testament (Acta Seminarii Neotestamentici Upsaliensis 20), Lund 1954.

Strack, H. L. - Billerbeck, P., Kommentar zum Neuen Testament aus Talmud und Midrasch. II, München 1924.

Valle, C. Del, La Misná. Segunda Edición Revisada y Corregida (Biblioteca de Estudios Bíblicos 98), Salamanca 2011.

VAn Tilborg, S., The Jewish Leaders in Matthew, Leiden 1972.

Viviano, B. T., "Evangelio según Mateo", en R. E. Brown - J. A. FitzmyerR. E. Murphy (eds.), Nuevo Comentario Bíblico San Jerónimo. II, Madrid 2004, 66-132. 
Weren, W. J. C., “The Macrostructure of Matthew's Gospel: A New Proposal", Bib 87 (2006) 171-200.

Westcott, B. F. - Hort, F. J. A., The New Testament in the Original Greek. I, Cambridge - London 1881.

WILLs, L. M., "Scribal Methods in Matthew and Mishnah Abot", CBQ 63 (2001) 241-257.

ZAHN, T., Das Evangelium des Matthäus (Kommentar zum Neuen Testament 1), Leipzig ${ }^{4} 1922$.

Zerwick, M., El Griego del Nuevo Testamento (Instrumentos para el Estudio de la Biblia 2), Estella ${ }^{3} 2002$.

Zerwick, M. - Grosvenor, M., Análisis gramatical griego del Nuevo Testamento, Estella 2008.

[recibido: 14/08/19 - aceptado: 29/10/19] 